\title{
Factores Asociados a la Implicación en Bullying: Un Estudio en Nicaragua
}

\section{Factors Associated with Involvement in Bullying: A Study in Nicaragua}

\author{
Eva M. Romera ${ }^{1}$, Rosario Del Rey ${ }^{2}$ y Rosario Ortega ${ }^{1}$ \\ ${ }^{1}$ Universidad de Córdoba, Spain \\ ${ }^{2}$ Universidad de Sevilla, Spain
}

\begin{abstract}
Resumen. El bullying escolar es uno de los principales problemas que afecta a la calidad de las relaciones interpersonales entre iguales en las escuelas y en general a la calidad de la convivencia. A nivel europeo, existen resultados científicos que indican su naturaleza, características y factores relacionados con su implicación. Sin embargo, en los países pobres y en desarrollo, donde el problema es aún más grave, hay un alto grado de desconocimiento de este problema. El presente estudio tiene como objetivo conocer qué factores pueden estar influyendo en la aparición de la intimidación en una muestra representativa de escuelas primarias en Managua y su área metropolitana. Para ello se ha entrevistado a 3042 niños y niñas que asisten a las escuelas primarias de la capital nicaragüense utilizando instrumentos similares a los utilizados en Europa. Un análisis de regresión logística multinomial señala que ser chico, manifestar conductas y actitudes antisociales y mantener contacto con drogas son tres factores relacionados con el rol de agresor. Relaciones interpersonales negativas ejercen una influencia significativa sobre la implicación en este fenómeno, ya sea como víctima, agresor o agresor victimizado. Los resultados se discuten en relación a los perfiles y figuras del agresor y víctima de bullying en estudios internacionales focalizando las diferencias respecto de países desarrollados.
\end{abstract}

Palabras clave: bullying, Nicaragua, países pobres, relaciones interpersonales.

\begin{abstract}
School bullying is one of the main problems affecting the quality of peer relationships in schools and in general the coexistence. At European level, there are scientific findings that indicate its nature, characteristics and factors related to its involvement. However, in poor and developing countries, where the problem is more serious, there is a high degree of awareness on this matter. The present study aimed to identify what factors may be influencing the occurrence of bullying in a representative sample of primary schools in the Nicaraguan capital. For this propose, 3042 students from Managua and its metropolitan area were explored with instruments comparable to those used in Europe. A multinomial logistic regression analysis indicated that being a boy, to show antisocial behaviors and attitudes and contact with drugs were the three factors most related with the role of aggressor, as well as negative relationships had a significant influence on involvement in this phenomenon, either as a victim, aggressor or victimized aggressor. Results are discussed in relation to the profiles of aggressor and victim of bullying in international studies focusing on differences in developed countries.
\end{abstract}

Keywords: bullying, interpersonal relationships, Nicaragua, poor countries.

Existe una preocupación internacional cada vez más generalizada por la violencia escolar, sus múltiples manifestaciones y la complejidad de su afrontamiento efectivo (Moore, Jones y Broadbent, 2008). A nivel europeo, las investigaciones sobre violencia escolar se han centrado particularmente en un tipo específico, el acoso y maltrato entre iguales, fenómeno conocido internacionalmente como bullying (Debarbieux y Blaya, 2001; Del Barrio, Martín, Montero, Gutiérrez, Barrios y De Dios, 2008; Díaz-Aguado, 2010; Ortega y Mora-Merchán, 2008; Smith, Morita, Junger-Tas,

Correspondencia: Eva M. Romera, Facultad de Ciencias de la Educación. Avda. San Alberto Magno, s/n., 14071. Córdoba. Spain. E-mail:m82rofee@uco.es
Olweus, Catalano y Slee, 1999). Gracias a ellas, hoy sabemos que suele estar presente en todas las escuelas y que afecta no solo a la calidad de las relaciones interpersonales y a la convivencia escolar, sino también al propio desarrollo de los escolares directamente implicados (Garner y Lemerise, 2007; Nabuzoka, Rønning y Handegård, 2009; Ortega, 2010).

A partir de los pioneros estudios escandinavos sobre el bullying (Olweus 1978; 1980; 1993) se ha desplegado un importante trabajo científico en todos los países del llamado mundo desarrollado (Jimerson, Swearer y Espelage, 2010; Smith y Brain, 2000). Fenómeno que fue descrito en un primer momento como acoso reiterado de uno o más estudiantes hacia otro/s mediante acciones negativas (Olweus, 1978) y que, posterior- 
mente, el propio Olweus y otros autores complementaron (Olweus, 1999; Ortega y Mora-Merchán, 2000; Rigby, 2002; Smith y Sharp, 1994). Se establecen tres características que definen el bullying: ser un comportamiento agresivo intencionado, repetición en el tiempo y desequilibrio de poder o fuerza entre sus protagonistas (Olweus, 2007). Adicionalmente, se han descrito dos leyes que posibilitan que el bullying se mantenga: la ley del silencio y la ley del dominio-sumisión. Por un lado, las personas implicadas, directa o indirectamente, tienden a ocultar lo que está sucediendo, lo que dificulta la detección del problema; y por otro, el mantenimiento entre la víctima y el agresor del vínculo en un insano juego de dominio-sumisión (Ortega, 2010).

Las manifestaciones del bullying son de naturaleza diversa, marcada por las conductas concretas que se activan: verbal (insultos, motes, etc.), física (golpes, patadas, puñetazos, etc.), psicológica (chantajes, amenazas, etc.) y relacional: exclusión social o aislamiento de la víctima ignorándola y excluyéndola (Wang, Iannotti y Nansel, 2009).

El análisis de la implicación en los problemas de bullying en un primer momento se realizó atendiendo a dos perfiles: víctimas y agresores (Olweus, 1978) e incluso un tercero mixto, de agresores victimizados o víctimas agresivas (Ortega, 2010; Rodkin y Berger, 2008; Schwartz, Dodge y Coie, 1993). Tipología que se ha ido enriqueciendo matizando la complejidad social del fenómeno (Salmivalli, Lagerspetz, Björkqvist, Österman y Kaukiainen, 1996), pero que ha sido escasamente considerada en los estudios que analizan variables relacionadas con la implicación.

Una de las líneas de investigación sobre el fenómeno se ha centrado en conocer y analizar los factores relacionados con la implicación directa de los y las escolares, ya sea atendiendo a factores individuales (Smith, 2009), del contexto familiar (Baldry y Farrington, 1998; Cava, Musitu, Buelga y Murgui, 2010; Povedano, Hendry, Ramos y Varela, 2011) o de las relaciones interpersonales que se establecen en el centro educativo, principalmente con el grupo de iguales (Dijkstra, Lindenberg y Veenstra, 2008; Duffy y Nesdale, 2009; Nesdale, Durkin, Maass, Kiesner y Griffiths, 2008).

En relación al contexto escolar, la implicación se relaciona con la percepción de un clima escolar desfavorable y una falta de apoyo por parte de los adultos (Barboza, Schiamberg, Oehmke, Korzeniewski y Post, 2009). En cuanto a los iguales, las víctimas y víctimas agresivas suelen ser escolares que se perciben, en mayor medida que los demás, con malas relaciones con sus compañeros (Cava et al., 2010; Ortega y MoraMerchán, 2008), mientras los agresores de bullying creen tener buena reputación y se perciben socialmente populares (Buelga, Musitu, Murgui y Pons, 2008). De hecho, en contra de lo que se solía creer, no son necesariamente sujetos socialmente rechazados (Rodkin y Berger, 2008).
Respecto del contexto familiar, se ha descrito que víctimas y agresores de bullying son sometidos, en mayor medida que otros menores, a un trato extremadamente coercitivo y hostil o están expuestos a contemplar conflictos o violencia adulta, aunque no siempre sean agredidos directamente en el seno familiar (Bowes, Arseneault, Maughan, Taylor, Caspi y Moffitt, 2009; Ferguson, San Miguel y Hartley, 2009). Ya en los años ochenta se puso de manifiesto que la exposición a modelos violentos incrementa el riesgo de aprendizaje vicario de la violencia (Bandura y Ribes, 1980). Otros estudios demuestran que el haber sido víctima de adultos se relaciona con la implicación en actos violentos, bien como agresor, reproduciendo el esquema violento en las relaciones con sus iguales, bien como víctima, aprendiendo a desarrollar una conducta sumisa ante el abuso de poder de los otros (Coyne y Monks, 2011).

En cuanto a la personalidad y las condiciones individuales que se relacionan con la implicación en bullying, destacan el sexo, la edad, las características físicas, la autoestima, la empatía, el éxito académico y muy particularmente la agresividad del sujeto (Farrington, 2005; Ortega, 2008). Los agresores suelen ser chicos y chicas que muestran impulsos socialmente dominantes y una actitud positiva hacia el uso de la violencia (Hunter, Mora-Merchán y Ortega, 2004; McConville y Cornell, 2003; Olweus y Endresen, 1998; Rutter, Giller y Hagell, 2000). Ser víctima de iguales se relaciona con niveles bajos de competencia social y baja autoestima (Cassidy, 2009). En cuanto al rol de las víctimas agresivas o agresores victimizados, Andreou (2000) señala que son chicos y chicas que presentan síntomas de conducta externalizante, tales como conducta inadaptada o pre-delincuente, así como algunos síntomas internalizantes como ansiedad y tendencia a la depresión.

Determinadas creencias, actitudes, valores y conductas, aunque no sean en sí mismos hechos violentos, también pueden estar relacionados con la tendencia a implicarse en fenómenos de bullying. Entre ellas se han descrito el consumo de drogas (Stein, Dukes y Warren, 2007; Tharp-Taylor, Haviland y D'Amico, 2009) y llevar armas (Nansel, Overpeck, Haynie, Ruan y Scheidt, 2003).

A pesar de este conocimiento preciso sobre los factores relacionados con la implicación de los y las escolares que se ven envueltos en fenómenos de bullying, aún quedan interrogantes al respecto. Uno de ellos es analizarlo en contextos que no pertenezcan al llamado mundo occidental, ya que podría suceder que los hallazgos encontrados con muestras de países desarrollados no sean necesariamente coincidentes con los que podrían hallarse en poblaciones de países pobres y en vías de desarrollo, donde algunos estudios han puesto de manifiesto que factores como la pobreza o la desigualdad social pueden perturbar el equilibrio en la relación social y dañar la calidad de vida, produciéndo- 
se una mayor tendencia al uso de la violencia (Del Rey y Ortega, 2008; Krug, Dahlberg, Mercy, Zwi y Lozano, 2002). Por tanto, se necesitan estudios que analicen los factores que están relacionados con la implicación en bullying en países pobres, como por ejemplo, es el caso de Nicaragua (Informe de la OECD-FAO, 2010). Pretensión que abordamos en la presente investigación.

El estudio que aquí se presenta explora las posibles relaciones entre la implicación en bullying y factores individuales, familiares y escolares en alumnado de educación primaria de Managua y su área metropolitana. La hipótesis desde la que se parte es que a pesar de las diferencias entre ambos tipos de sociedades, los factores relacionados con la implicación son similares.

\section{Metodología}

\section{Participantes}

La muestra representativa estuvo compuesta por 3042 estudiantes (50.3\% niñas) de los tres últimos cursos presenciales de Educación Primaria $\left(4^{\circ}, 5^{\circ}\right.$ y $6^{\circ}$, correspondientes teóricamente a edades entre 10 y 12 años) de Managua y su área metropolitana. Las edades de los sujetos estaban comprendidas entre los 8 y los 22 años $(M=11.3 ; D T=1.6)$, por lo que se establecieron cinco tramos de edad para el posterior análisis: escolares de 9 años o menos (11.81\%), 10 años (20.82\%), 11 años (26.51\%), 12 años $(19.88 \%)$ y 13 años o más $(20.98 \%)$.

\section{Instrumento}

El Instrumento utilizado fue el Cuestionario sobre Convivencia, Violencia y Experiencias de RiesgoCOVER (Ortega y Del Rey, 2003), elaborado ad hoc con el objetivo de establecer una línea base para el diseño de políticas preventivas ante la problemática de los escolares de Educación Primaria de Nicaragua. Está compuesto por 54 ítems de tipo Likert 1-3 (grado de acuerdo y frecuencia) y el tiempo de referencia utilizado alude a los tres últimos meses. Tras un estudio piloto en el que se utilizó una muestra de 429 alumnos y alumnas y una validación de expertos (expertos internacionales sobre bullying, expertos nacionales sobre políticas sociales y educativas y jóvenes nacionales), se realizó un análisis multivariante Homals, recomendado para variables no cuantitativas (Visauta y Martori, 2003), en el que se encontraron nueve escalas que fueron agrupadas en tres dimensiones: Valor personal y sociabilidad: a) autopercepción y valoración personal (autovalor $=.78)$ (4 ítems; ej.: "he pensado que no valgo nada"); b) afrontamiento de problemas y sociabilidad (.60) (4 ítems; ej.: "si me invitan a tomar me veo obligado a tomar"); c) relaciones interpersonales (.78) (3 ítems; ej.: "mis profesores se han portado bien conmigo y me han ayudado"). Actitudes y conductas de riesgo: a) comportamientos y actitudes antisociales (.84) (5 ítems; ej.: "no me relaciono con gente que considero inferior"); b) contexto social violento (.69) (5 ítems; ej.: "mi escuela es más violenta que las demás"); c) consumo y tráfico de drogas (.81) (4 ítems; ej.: "he consumido algún tipo de droga"). Implicación directa en violencia: a) victimización de iguales (.83) (8 ítems; ej.: "algún compañero/a me ha insultado"); b) maltrato y abuso de adultos hacia escolares (.71) (4 ítems; ej.: "algún maestro/a me ha golpeado"); y c) comportamiento agresivo (.75) (6 ítems; ej.: "he golpeado a algún compañero/a").

\section{Procedimiento}

La muestra fue seleccionada mediante muestreo aleatorio estratificado con un tipo de afijación proporcional (Alba y Ruiz, 2004) conforme a los criterios ubicación (los seis distritos de Managua más su área metropolitana) y modo de financiación del centro escolar (público, privado o subvencionado). El tamaño de la muestra se distribuyó de forma proporcional (5\%) al de la presencia de cada estrato en la población.

Desde el Ministerio de Educación, Cultura y Deportes de Nicaragua se solicitó a los centros seleccionados la participación en la investigación. Una vez hubieron aceptado, miembros del equipo de investigación e investigadores entrenados a tal efecto, se desplazaron a los mismos a administrar los cuestionarios durante el horario de clase en ausencia del profesorado. Antes de completar el cuestionario se enfatizó al alumnado la naturaleza voluntaria y anónima de la participación y la importancia de contestar de forma honesta.

En cuanto a la codificación, la implicación en bullying se estimó a partir de las escalas victimización de iguales y comportamiento agresivo, considerando opción de respuesta muchas veces en cualquiera de las manifestaciones de violencia entre iguales (física, verbal, psicológica o relacional), definiéndose cuatro roles de implicación: agresor, víctima, agresor victimizado y espectador (Schwartz, Dodge, Pettit y Bates, 1997). En la tabla 1 se resume este criterio de selección. El resto de escalas fueron recodificadas en una variable cada una para su posterior análisis siguiendo un proceso homólogo en todos los casos (Silva y Barroso, 2004). Dicha recodificación consistió en dicotomizar los valores de las variables (ausencia presencia) utilizando un criterio exigente para ello, ya que solo se incluyó en valor de presencia la opción máxima cuando se refiriese a aspectos negativos y mínima cuando fueran positivos, a excepción de los indicadores relativos a la autopercepción y valoración personal, a las actitudes y conductas antisociales y al contacto con drogas, cuyo valor final es el resultado del sumatorio de las puntuaciones. 
Tabla 1. Criterio de selección de los implicados en bullying

\begin{tabular}{llll}
\hline & $\begin{array}{l}\text { Agresor } \\
\text { Nunca }\end{array}$ & $\begin{array}{l}\text { Agresor } \\
\text { Pocas }\end{array}$ & $\begin{array}{l}\text { Agresor } \\
\text { Muchas }\end{array}$ \\
\hline Víctima nunca & Espectador & Espectador & Agresor \\
Víctima pocas & $\begin{array}{l}\text { Espectador } \\
\text { Víctima muchas }\end{array}$ & $\begin{array}{l}\text { Eśctima } \\
\text { Víctima }\end{array}$ & $\begin{array}{l}\text { Agresor } \\
\text { Agresor victimizado }\end{array}$ \\
\hline
\end{tabular}

\section{Análisis de datos}

Se realizaron dos tipos de análisis. En primer lugar, un análisis univariado para evaluar la relación entre la implicación en bullying y cada una de las variables independientes. En este caso se realizaron contrastes de proporciones $\left(\chi^{2}\right)$, tomando de referencia los residuos tipificados corregidos (valores superiores a 1.96 para un intervalo de confianza del $95 \%$ y superiores a 2.58 para el 99\%) y contrastes de medias (análisis de varianza, ANOVA) utilizando la prueba de Bonferroni. Aquellas variables que resultaron significativas fueron incluidas en un análisis multivariado de regresión logística multinomial.

Los análisis fueron realizados con el paquete estadístico SPSS versión 15.0 para Windows. El nivel de significatividad adoptado fue de $p<.05$.

\section{Resultados}

La implicación en bullying resultó del 6\% agresores, el $25.3 \%$ víctimas, el $18.7 \%$ agresor victimizado y el $50 \%$ espectadores.

\section{Relaciones con la implicación en bullying: análisis univariado}

Los resultados mostraron que existían diferencias estadísticamente significativas entre todas las variables introducidas en el análisis y la implicación en bullying (ver tabla 2). En cuanto al sexo, los agresores victimizados solían ser chicos y los espectadores tendían a ser chicas, $\chi^{2}(3, n=2440)=39.393, V=.127 ; p=.000$. En el análisis de las relaciones entre escolares eran los agresores victimizados quienes más relaciones negativas mantenían con sus iguales, siendo los espectadores quienes menos, $\chi^{2}(3, n=2367)=17.392, V=.086$; $p<.01$. En las relaciones de los estudiantes con sus docentes los espectadores fueron los que emitieron más respuestas positivas, $\chi^{2}(3, n=2384)=18.891, V$ $=.089 ; p=.000$. Tanto víctimas como agresores victimizados percibían una mayor tendencia de sus familias a ir a hablar con los adultos responsables en la escuela y las familias de los espectadores las que menos contacto establecían, $\chi^{2}(3, n=2450)=57.156, V=.153$; $p=.000$. En relación al contexto social violento eran los agresores victimizados los que en mayor medida estaban presentes en múltiples contextos violentos, $\chi^{2}$ $(9, n=2184)=132.533, V=.142 ; p=.000$. Del mismo modo, eran los agresores victimizados los que afirmaban en mayor medida ser víctimas también de maltrato de sus progenitores o docentes, seguidos de las víctimas (20\%), mientras que los espectadores confirmaron de manera más notoria no haber sido víctimas de este tipo de maltrato, $\chi^{2}(3, n=2373)=322.101, V=$ $.368 ; p=.000$. Por último, los agresores victimizados reconocieron en mayor medida ser espectadores de

Tabla 2. Valores y porcentajes de las pruebas Chi Cuadrado

\begin{tabular}{|c|c|c|c|c|c|}
\hline Sexo & Valores & A & $\mathrm{V}$ & AV & $\mathrm{E}$ \\
\hline & $\mathrm{H}$ & 55.1 & 49.2 & $62.9 * *$ & $46^{* *}$ \\
\hline & M & 44.9 & 50.8 & $37.1 * *$ & $54 * *$ \\
\hline Relaciones iguales & $\begin{array}{l}- \\
+\end{array}$ & $\begin{array}{l}14.5 \\
85.5\end{array}$ & $\begin{array}{l}14.8 \\
85.2\end{array}$ & $\begin{array}{l}18.5^{* *} \\
81.5^{* *}\end{array}$ & $\begin{array}{l}10.8^{* *} \\
89.2^{* * *}\end{array}$ \\
\hline Relaciones alumno/docente & - & $\begin{array}{l}20.9 \\
79.1\end{array}$ & $\begin{array}{l}17.2 \\
82.8\end{array}$ & $\begin{array}{l}26.9 * * \\
73.1 * *\end{array}$ & $\begin{array}{l}17.9^{*} \\
82.1^{*}\end{array}$ \\
\hline Relaciones familia/escuela & - & $\begin{array}{l}31.6 \\
68.4\end{array}$ & $\begin{array}{l}26.3^{* *} \\
73.7^{* *}\end{array}$ & $\begin{array}{l}22.8^{* *} \\
77.2^{* *}\end{array}$ & $\begin{array}{l}39.4^{* *} \\
60.6^{* *}\end{array}$ \\
\hline Contexto social violento & $\begin{array}{l}0 \\
1 \\
2 \\
3\end{array}$ & $\begin{array}{l}19.7 \\
40.1 \\
29.9 \\
10.2\end{array}$ & $\begin{array}{l}19.7^{* *} \\
39.9 \\
28.1 \\
12.4^{*}\end{array}$ & $\begin{array}{l}11.3^{* *} \\
36.4^{*} \\
34.6^{* *} \\
17.7^{* *}\end{array}$ & $\begin{array}{c}30.8^{* *} \\
43.5^{*} \\
19.9^{* *} \\
5.8^{* *}\end{array}$ \\
\hline Victimización adultos & $\begin{array}{l}\text { No } \\
\text { Sí }\end{array}$ & $\begin{array}{l}88.8 \\
11.2\end{array}$ & $\begin{array}{l}80.8^{* *} \\
19.2 * *\end{array}$ & $\begin{array}{l}60.6^{* *} \\
39.4^{* *}\end{array}$ & $\begin{array}{r}95.7 * * \\
4.3 * *\end{array}$ \\
\hline Testigo peleas violentas & $\begin{array}{l}\text { No } \\
\text { Sí }\end{array}$ & $\begin{array}{l}46.2^{*} \\
53.8^{*}\end{array}$ & $\begin{array}{l}44.7^{* *} \\
55.3^{* *}\end{array}$ & $\begin{array}{l}31.6^{* *} \\
68.4^{* *}\end{array}$ & $\begin{array}{l}70.6^{* *} \\
29.4^{* *}\end{array}$ \\
\hline
\end{tabular}

* Residuos tipificados corregidos con valores superiores a \pm 1.96 (intervalo de confianza $95 \%$ )

** Residuos tipificados corregidos con valores superiores a \pm 2.58 (intervalo de confianza $99 \%$ ) 
violencia frente a los no implicados, que manifestaron no haber presenciado acciones violentas, $\chi^{2}(3, N=$ $2442)=251.071, V=.321 ; p=.000$.

Diferencias estadísticamente significativas fueron observadas en relación al indicador de autopercepción y valoración personal, $F(3, N=2150)=34.841 ; p=$ .000. Los resultados obtenidos en la prueba Post-hoc mostraron que estas diferencias significativas se registraron principalmente en los espectadores y en los agresores victimizados, siendo los primeros los que mostraron el nivel más alto en autopercepción y valoración personal y los segundos los que presentaron el nivel más bajo de los cuatro roles estudiados (véase tabla 3 ).

Resultados similares se observaron en actitudes y conductas antisociales, $F(3, N=2215)=92.335, p=$ .00 y contacto con drogas, $F(3, N=2354)=36.737$, $p=.00$. Las diferencias estadísticamente significativas indicaban que eran los agresores victimizados los que mayor número de actitudes y comportamientos violentos manifestaban y además eran los que mantenían un mayor contacto con las drogas frente a los espectadores, que eran quienes menos manifestaban este tipo de actitudes y conductas (véase tabla 3). La edad no resultó significativa.

\section{Implicación en bullying: análisis multivariado}

En este análisis se incluyeron todas las variables que mostraron diferencias estadísticamente significativas en el análisis univariado. Para introducir las variables en la ecuación de regresión se utilizó el método por pasos hacia delante.
El análisis de regresión logística resultó significativo para las siguientes nueve variables: sexo, testigo de peleas violentas, autopercepción y valoración personal, relaciones entre iguales, relaciones con profesores, relaciones entre familia y profesorado, víctima de adultos, contacto con drogas y actitudes y conductas antisociales, -2LL $=2359,918 ; \chi^{2}(27, N=3042)=$ $525.924, p=.00$. Este modelo explicó el $35.6 \%$ de los casos. La categoría de referencia fue el espectador.

\section{El agresor de bullying}

Los resultados indicaron que existía una fuerte relación entre el perfil de agresor y las relaciones negativas con los iguales, el contacto con drogas, la manifestación de actitudes y comportamientos antisociales, así como el ser chico. También influía, aunque en menor grado, una baja autopercepción y valoración personal, el que los padres y madres tuvieran malas relaciones con los docentes y, en última instancia, el que se hubiera presenciado peleas violentas (ver tabla 4).

\section{La víctima de bullying}

La variable que más se relaciona con el perfil de víctima de bullying es el hecho de tener malas relaciones con los compañeros y compañeras, seguido de una autopercepción y valoración personal negativas. También se observaron relaciones importantes, aunque en menor medida, entre la victimización y las malas relaciones entre familias y docentes, seguido de haber sido

Tabla 3. Media de las variables cuantitativas (desviación típica)

\begin{tabular}{|c|c|c|c|}
\hline \multirow[t]{2}{*}{ Actitudes y conductas antisociales } & \multirow[t]{2}{*}{ Media (d. t.) } & \multicolumn{2}{|c|}{ Media (d. t.) } \\
\hline & & Chico & Chica \\
\hline Agresor & $0.89(.850)^{\mathrm{av}, \mathrm{e}}$ & $1.03(.833)$ & $0.73(.863)$ \\
\hline Víctima & $0.75(.813)^{\text {av, e }}$ & $0.81(.807)$ & $0.67(.802)$ \\
\hline Agresor victimizado & $1.34(.961)^{\mathrm{a}, \mathrm{v}, \mathrm{e}}$ & $1.37(1.000)$ & $1.23(.856)$ \\
\hline Espectador & $0.55(.741)^{\mathrm{a}, \mathrm{v}, \mathrm{av}}$ & $0.58(.741)$ & $0.51(.738)$ \\
\hline \multirow[t]{2}{*}{ Autopercepción y valoración personal } & Media (d. t.) & \multicolumn{2}{|c|}{ Media (d. t.) } \\
\hline & & Chico & Chica \\
\hline Agresor & $6.68(1.815)^{\text {av, e }}$ & $6.69(1.990)$ & $6.65(1.620)$ \\
\hline Víctima & $6.87(1.685)^{\mathrm{av}, \mathrm{e}}$ & $6.80(1.707)$ & $6.93(1.684)$ \\
\hline Agresor victimizado & $6.23(1.914)^{\mathrm{a}, \mathrm{v}, \mathrm{e}}$ & $6.35(1.961)$ & $6.05(1.863)$ \\
\hline Espectador & $7.23(1.561)^{\mathrm{a}, \mathrm{v}, \mathrm{av}}$ & $7.23(1.511)$ & $7.21(1.605)$ \\
\hline \multirow[t]{2}{*}{ Contacto con drogas } & Media (d. t.) & \multicolumn{2}{|c|}{ Media (d. t.) } \\
\hline & & Chico & Chica \\
\hline Agresor & $0.27(.460)^{\text {av, e }}$ & $0.26(.443)$ & $0.24(.442)$ \\
\hline Víctima & $0.22(.438)^{a v, e}$ & $0.24(.465)$ & $0.20(.412)$ \\
\hline Agresor victimizado & $0.39(.611)^{\mathrm{a}, \mathrm{v}, \mathrm{e}}$ & $0.41(.649)$ & $0.35(.547)$ \\
\hline Espectador & $0.14(.342)^{\mathrm{a}, \mathrm{v}, \mathrm{av}}$ & $0.12(.322)$ & $0.14(.350)$ \\
\hline
\end{tabular}

a, v, av, e : siglas de los roles que indican entre qué medias existen diferencias significativas 
Tabla 4. Regresión logística para el perfil de agresor de bullying

\begin{tabular}{|c|c|c|c|c|c|}
\hline & \multirow[t]{2}{*}{$\beta$} & \multirow[t]{2}{*}{ Wald } & \multirow[t]{2}{*}{$\operatorname{Exp}(\beta) O R$} & \multicolumn{2}{|c|}{ I. C. al $95 \%$ para $\operatorname{Exp}(\beta)$} \\
\hline & & & & L. Inferior & L. Superior \\
\hline \multicolumn{6}{|l|}{ Sexo } \\
\hline Hombre & 0.510 & 4.417 & $1.665^{*}$ & 1.035 & 2.679 \\
\hline Mujer & -0.510 & & & & \\
\hline Testigo violencia & 0.785 & 10.186 & $0.456^{*}$ & 0.281 & 0.739 \\
\hline Rel. con iguales negativas & 0.872 & 6.528 & $2.392 *$ & 1.225 & 4.670 \\
\hline Rel. con profes. negativas & 0.356 & 1.441 & 1.427 & 0.799 & 2.551 \\
\hline Rel. pad.-prof. negativas & -0.586 & 4.770 & $0.557 *$ & 0.329 & 0.942 \\
\hline Víctima adultos & 0.362 & 0.618 & 0.696 & 0.282 & 1.717 \\
\hline Autopercepción valor. personal & -0.257 & 13.172 & $0.774 *$ & 0.674 & 0.889 \\
\hline Act. y cond. antisociales & 0.574 & 15.755 & $1.775^{*}$ & 1.337 & 2.357 \\
\hline Contacto con drogas & 0.675 & 6.717 & $1.964 *$ & 1.179 & 3.271 \\
\hline
\end{tabular}

*Resultados significativos menores que .05

víctima de adultos, así como testigo de peleas violentas (ver tabla 5).

\section{El agresor victimizado de bullying}

En cuanto al rol del agresor victimizado, los nueve indicadores de factores de riesgo guardaban relación con este perfil, destacando principalmente el ser chico, tener malas relaciones interpersonales con los compañeros y compañeras, mostrar actitudes y conductas antisociales, tener contacto con drogas y mantener malas relaciones con el profesorado. En menor medida, también influían el bajo nivel de autopercepción y valoración personal, las malas relaciones entre familia y docentes, así como el haber sido testigo de peleas violentas (ver tabla 6).

\section{Conclusiones y discusión}

Este estudio partía del objetivo de explorar las posibles relaciones entre la implicación en bullying y fac-

Tabla 5. Resultados de la regresión para las víctimas de bullying tores individuales, familiares y escolares en alumnado de Educación Primaria de Managua y su área metropolitana. Los resultados nos permiten concluir que los factores relacionados con la implicación en bullying en la muestra de estudio son similares a las sociedades desarrolladas, lo cual apoya nuestra hipótesis de partida.

El bullying severo está presente en las escuelas de Managua y su área metropolitana a un nivel elevado. Cifras que no deben compararse con estudios realizados en los que la implicación se analiza a partir de respuestas directas y sin criterios excluyentes de selección a la hora de definir la implicación (Defensor del Pueblo, 2000; 2007). Es decir, la capital de Nicaragua y su área metropolitana, además de ser un lugar aquejado de serios problemas económicos y sociales, resulta tener, en su sistema educativo básico, la Educación Primaria, problemas de maltrato entre escolares; sin embargo, no hay en su sociedad conciencia de que el bullying sea un verdadero problema escolar (Ortega, 2003).

Tener malas relaciones interpersonales entre el grupo de iguales es un factor de riesgo de que acontez-

\begin{tabular}{|c|c|c|c|c|c|}
\hline & \multirow[t]{2}{*}{$\beta$} & \multirow[t]{2}{*}{ Wald } & \multirow[t]{2}{*}{$\operatorname{Exp}(\beta) O R$} & \multicolumn{2}{|c|}{ I. C. al $95 \%$ para $\operatorname{Exp}(\beta)$} \\
\hline & & & & L. Inferior & L. Superior \\
\hline \multicolumn{6}{|l|}{ Sexo } \\
\hline Hombre & 0.118 & 0.680 & 1.125 & 0.850 & 1.490 \\
\hline Mujer & -0.118 & & & & \\
\hline Testigo violencia & 1.023 & 48.843 & $0.360^{*}$ & 0.270 & 0.479 \\
\hline Rel. con iguales negativas & 0.588 & 6.395 & $1.801 *$ & 1.142 & 2.842 \\
\hline Rel. con profes. negativas & -0.086 & 0.187 & 0.917 & 0.621 & 1.356 \\
\hline Rel. pad.-prof. negativas & -0.687 & 18.286 & $0.503 *$ & 0.367 & 0.689 \\
\hline Víctima adultos & 1.645 & 40.960 & $0.193 *$ & 0.117 & 0.320 \\
\hline Autopercepción valor. personal & -0.152 & 11.715 & $0.859 *$ & 0.788 & 0.937 \\
\hline Act. y cond. antisociales & 0.182 & 3.408 & 1.199 & 0.989 & 1.454 \\
\hline Contacto con drogas & -0.016 & 0.007 & 0.984 & 0.672 & 1.441 \\
\hline
\end{tabular}

*Resultados significativos menores que .05 
Tabla 6. Regresión logística para el rol de agresor victimizado

\begin{tabular}{|c|c|c|c|c|c|}
\hline & \multirow[t]{2}{*}{$\beta$} & \multirow[t]{2}{*}{ Wald } & \multirow[t]{2}{*}{$\operatorname{Exp}(\beta) O R$} & \multicolumn{2}{|c|}{ I. C. al $95 \%$ para $\operatorname{Exp}(\beta)$} \\
\hline & & & & L. Inferior & L. Superior \\
\hline \multicolumn{6}{|l|}{ Sexo } \\
\hline Hombre & 0.815 & 18.088 & $2.260^{*}$ & 1.552 & 3.291 \\
\hline Mujer & -0.815 & & & & \\
\hline Testigo violencia & 1.438 & 52.218 & $0.237^{*}$ & 0.161 & 0.351 \\
\hline Rel. con iguales negativas & 0.791 & 7.956 & $2.206^{*}$ & 1.273 & 3.822 \\
\hline Rel. con profes. negativas & 0.657 & 8.551 & $1.928^{*}$ & 1.242 & 2.994 \\
\hline Rel. pad.-prof. negativas & -0.734 & 11.870 & $0.480^{*}$ & 0.316 & 0.729 \\
\hline Víctima adultos & 1.923 & 47.382 & $0.146^{*}$ & 0.085 & 0.253 \\
\hline Autopercepción valor. personal & -0.297 & 29.279 & $0.743^{*}$ & 0.667 & 0.827 \\
\hline Act. y cond. antisociales & 0.930 & 67.817 & $2.536^{*}$ & 2.032 & 3.164 \\
\hline Contacto con drogas & 0.699 & 11.843 & $2.011 *$ & 1.351 & 2.995 \\
\hline
\end{tabular}

*Resultados significativos menores que .05

can abusos de poder, intimidación, y en general malos tratos (Dijkstra et al., 2008; Duffy y Nesdale, 2009; Ortega y Mora-Merchán, 2008); precisamente, en las escuelas de primaria de Managua, éste es un factor que implica un serio riesgo de verse implicado en bullying sea como víctima o como agresor o como ambos roles a la vez. Así lo confirman los resultados, en los que tener malas relaciones con los compañeros y compañeras aumenta la probabilidad de verse implicado en fenómenos de bullying, en cualquiera de los tres roles en los que se ha basado nuestro análisis; es difícil saber por qué, pero no cabe duda que, o bien el nivel de violencia al que han estado expuestos ha sobrepasado su capacidad de afrontamiento o quizás, se deba a un bajo nivel de competencia social, tal y como afirman Eslea et al. (2004), Pepler, Jiang, Craig y Connolly (2008) y Vaillancourt, Brendgen, Boivin y Tremblay (2003). Ambas hipótesis interpretativas son coherentes, ya que se puede afirmar que el sistema educativo nicaragüense, como gran parte de los sistemas educativos de la zona, es deficitario tanto en competencia profesional de los docentes como en recursos mínimos que permitan una atención básica a la formación afectivo-emocional (Ministerio de Educación de Nicaragua, 2008).

Tres factores parecen determinantes en el riesgo de verse implicado en bullying en Managua: ser chico, manifestar conductas y actitudes antisociales y mantener contacto con drogas. Tres variables claramente definitorias del rol de agresor y que son excluyentes para el rol de víctima. Esto es coherente con las tendencias generales de los estudios internacionales sobre bullying, que indican que los varones, mucho más que las chicas, se ven implicados en el rol de agresores o agresores victimizados (Defensor del Pueblo-UNICEF, 2007; Farrington, 2005; Kristensen y Smith, 2003; Ortega, 2010; Smith, 2009). Ciertas actitudes antisociales y comportamientos de riesgo, como portar armas, hacer uso de la violencia reactiva ante hechos no muy ofensivos, o justificar la violencia física por motivos de celos o infidelidad que, desde un punto de vista teórico han sido considerados de riesgo (Nansel et al., 2003; Stein et al., 2007), inciden también en la vulnerabilidad ante el bullying escolar en Managua y su área metropolitana. Estos factores, como el contacto con personas que trafican con droga o la implicación en el pequeño comercio de la droga, están relacionados con la conducta de los agresores escolares, pero fundamentalmente de los escolares denominados agresores victimizados o víctimas agresivas, mostrando así un mayor nivel de vulnerabilidad. Quizás podemos asumir que la pobreza y las malas condiciones de vida aumentan el riesgo de lo que se ha llamado el multivictimismo (Blaya, Debarbieux, Del Rey y Ortega, 2006).

Respecto a las variables de personalidad que hemos estudiado, se ha descrito que existe cierta tendencia que relaciona la baja autoestima como característica personal con ser víctimas de los compañeros y compañeras (Andreou, 2000; Cassidy, 2009). Nuestro estudio muestra que una baja valoración de sí mismo está presente no sólo entre las víctimas, sino en todos los escolares implicados en fenómenos de bullying escolar, cualquiera que sea el rol en el que lo hagan, independientemente de que sea ejecutor de la acción violenta, víctima de la misma o agresor victimizado, tal y como han encontrado Farrington y Baldry (2005) en poblaciones igualmente muy vulnerables.

Quizás se establece lo que Ohsako (1999) denomina una cultura de la violencia, en la que el abuso de poder, el sometimiento, la minusvaloración de uno mismo, el contexto violento y las experiencias padecidas, se extrapolan de la vida social en general al microcosmos que es la convivencia escolar. En un intento por explicar la posible relación entre contexto violento y bullying, el análisis de los datos refleja que la percepción directa de casos de agresión es lo que impacta en la implicación (Coyne y Monks, 2011). Por el contrario, el hecho de que perciban el entorno o los entornos en los que viven como negativos o violentos, no pare- 
ce estar relacionado con la implicación en un rol específico, remarcando así la importancia del factor presencial y visible del acto violento.

Este estudio ha puesto de manifiesto que el rol ambivalente (víctima agresiva o agresor-victimizado) tiene un gran protagonismo. Éste es un rol escasamente estudiado en las investigaciones internacionales, aunque sí se ha encontrado con una cierta entidad en los estudios hispanos (Del Rey y Ortega, 2008; Wang et al., 2009). Es difícil hipotetizar sobre su importancia, pero no cabe duda de que cuando en un microsistema de convivencia entre iguales está muy presente el rol bully-victim es muy posible que estemos ante un sistema en el que las normas y los valores no están lo suficientemente claros y a los escolares les resulte difícil asumir una posición frente a ellas. Esto es coherente con los déficits del sistema educativo de Nicaragua, que está a un nivel muy precario tanto en términos de recursos materiales como de competencia profesional docente (Ministerio de Educación de Nicaragua, 2008). El estudio que aquí se presenta parece reclamar que los organismos gubernamentales supranacionales y nacionales, así como los agentes de la actividad docente se impliquen en la mejora de la calidad de vida y la convivencia de los escolares, con especial atención a la red de iguales. Son necesarias estrategias educativas y de ayuda que fortalezcan esta red en la medida en que es uno de los factores más determinantes en la vulnerabilidad de los individuos y posiblemente el más protector.

Por último, matizar que los resultados obtenidos deben ser interpretados dentro de una serie de limitaciones. En primer lugar, aunque se cuenta con una amplia muestra, la población de referencia no fue extensible a todo el país, por lo que se debe ser cauteloso a la hora de generalizar estos resultados a la población. En segunda instancia, el propio instrumento ha planteado una serie de limitaciones en cuanto a las variables de estudio y su medida, lo que ha limitado las opciones de análisis. En tercer lugar, el tamaño muestral de algunos grupos se plantea como una limitación de los propios resultados obtenidos. En este sentido, serían necesarios estudios longitudinales para conocer el modo en que unas variables afectan o predicen otras. Finalmente, sería conveniente hacer extensible el uso de instrumentos homogéneos en países latinoamericanos con un contexto social más homologable.

\section{Agradecimientos}

Este estudio ha sido realizado gracias a la financiación de Cooperación Británica a través del Departament for International Development (DFID) y al Convenio de colaboración entre el Ministerio de Educación, Cultura y Deportes de Nicaragua y la Universidad de Córdoba.

\section{Referencias}

Alba, M. V. y Ruiz, N. (2004). Muestreo estadístico. Oviedo: Septem Ediciones.

Andreou, E. (2000). Bully/victim problems and their association with psychological constructs in 8 to 12 year-old Greek schoolchildren. Aggressive Behavior, 26, 49-56.

Baldry, A. C. y Farrington, D. P. (1998). Parenting influences on bullying and victimization. Legal and Criminological Psychology, 3, 237-254.

Bandura, A. y Ribes, E. (1980). Modificación de conducta. Análisis de la agresión y de la delincuencia. México: Trillas.

Barboza, G. E., Schiamberg, L. B., Oehmke, J., Korzeniewski, S. J., Post, L. A. y Heraux, C. G. (2009). Individual characteristics and the multiple contexts of adolescent bullying: An ecological perspective. Journal of Youth and Adolescence, 38, 101-121.

Blaya, C., Debarbieux, E., Del Rey, R. y Ortega, R. (2006). Clima y violencia escolar. Un estudio comparativo entre España y Francia. Revista de Educación, 339, 293-315.

Bowes, L., Arseneault, L., Maughan, B., Taylor, A., Caspi, A. y Moffitt, T. (2009). School, Neighborhood, and Family Factors Are Associated With Children's Bullying Involvement: A Nationally Representative Longitudinal Study. Journal of the American Academy of Child \& Adolescent Psychiatry, 48, 545-553.

Buelga, S., Musitu, G., Murgui, S. y Pons, J. (2008). Reputation, Loneliness, Satisfaction with Life and Aggressive Behavior in Adolescence. Spanish Journal of Psychology, 11, 192-200.

Cassidy, T. (2009). Bullying and victimization in school children: The role of social identity, problem-solving style, and family and school context. Social Psychology of Education, 12, 63-76.

Cava, M. J., Musitu, G., Buelga, S. y Murgui, S. (2010). The relationships of family and classroom enviroments with peer relational victimization: an analysis of their gender differences. Spanish Journal of Psychology, 13, 156-165.

Coyne, I. y Monks, C. (2011). An overview of bullying and abuse across settings. Cambridge: Cambridge University Press.

Debarbieux, E. y Blaya, C. (2001). Violence in schools. Ten approaches in Europe. Issy-les-Moulineaux: ESF.

Defensor del pueblo-UNICEF. (2000). Violencia escolar: el maltrato entre iguales en la Educación Secundaria Obligatoria. C. Del Barrio, E. Martín, I. Montero, L. Hierro, I. Fernández, H. Gutiérrez y E. Ochaíta. Madrid: Defensor del Pueblo.

Defensor del Pueblo-UNICEF. (2007). Violencia escolar: el maltrato entre iguales en la educación secundaria obligatoria 1999-2006. Nuevo estudio y actualización del informe del 2000. C. del Barrio, M. A. Espinosa, E. Martín y E. Ochaíta. Madrid: Defensor del Pueblo.

Del Barrio, C., Martín, E., Montero, I., Gutiérrez, H. Barrios, A. y De Dios, M. J. (2008). Bullying and social exclusion in Spanish secondary schools: National trends from 1999 to 2006. International Journal of Clinical and Health Psychology, 8, 657-677. 
Del Rey, R. y Ortega, R. (2008). Bullying en los países pobres: prevalencia y coexistencia con otras formas de violencia. International Journal of Psychology and Psychological Therapy, 8, 39-50.

Díaz-Aguado, M. J. (2010). Estudio estatal sobre la convivencia escolar en la educación secundaria obligatoria. Madrid: Observatorio Estatal de la Convivencia Escolar.

Dijkstra, J. K., Lindenberg, S. y Veenstra, R. (2008). Beyond the class norm: Bullying behavior of popular adolescents and its relation to peer acceptance and rejection. Journal of Abnormal Child Psychology, 36, 1289-1299.

Duffy, A. L. y Nesdale, D. (2009). Peer groups, social identity, and children's bullying behaviour. Social Development, 18, 121-139.

Eslea, M., Menesini, E., Morita, Y., O’Moore, M., MoraMerchán, J. A., Pereira, B., ... Wenxin, Z. (2004). Friendship and loneliness among bullies and victims: Data from seven countries. Aggressive Behavior, 30, 71-83.

Farrington, D. P. (2005). The importance of child and adolescent psychopathy. Journal of Abnormal Child Psychology, 33, 489.497.

Farrington, D. P. y Baldry, A. C. (2005). Factores de riesgo individuales de la violencia escolar. Trabajo presentado en el IX Encuentro Internacional Violencia y Escuela. Centro Reina Sofía para el Estudio de la Violencia, Valencia.

Ferguson, C. J., San Miguel, C. M. y Hartley, R. D. (2009). A Multivariate Analysis of Youth Violence and Aggression: The Influence of Family, Peers, Depression, and Media Violence. The Journal of Pediatrics, 155, 904908.

Garner, P. W. y Lemerise, E. A. (2007). The roles of behavioral adjustment and conceptions of peers and emotions in preschool children's peer victimization. Development and Psychopathology, 19, 57-71.

Hunter, S. C., Mora-Merchán, J. A. y Ortega, R. (2004). The Long-Term Effects of Coping-Strategy Use in the Victims of Bullying. Spanish Journal of Psychology, 7, 3-12.

Jimerson, S. R., Swearer, S. M. y Espelage, D. L. (2010). Handbook of Bullying in School. An International Perspective. Nueva York: Routlege.

Kristensen, S. M. y Smith, P. K. (2003). The use of coping strategies by Danish children classed as bullies, victims, bully/victims, and not involved, in response to different (hypothetical) types of bullying, Sacandinavian Journal of Psychology, 44, 479-488.

Krug, E. G., Dahlberg, L. L., Mercy, J. A., Zwi, A. y LozanoAscencio, R. (2002). Informe Mundial sobre la Violencia y la Salud. Ginebra: Organización Mundial de la Salud.

McConville, D. W. y Cornell, D. G. (2003). Aggressive attitudes predict aggressive behavior in middle school students. Journal of Emotional and Behavioral Disorders, $11,179-187$.

Ministerio de Educación de Nicaragua (2008). Sistema Estadístico Nacional (SEN). Managua: Dirección General de Planificación y División de Estadística.

Moore, K., Jones, N. y Broadbent, E. (2008). School Violence in OECD countries. Londres: Plan Limited.
Nabuzoka, D., Rønning, J.A. y Handegård, B.H. (2009). Exposure to bullying, reactions and psychological adjustment of secondary school students. Educational Psychology, 7, 849-866.

Nansel, T. R., Overpeck, M. D., Haynie, D. L., Ruan, W. J. y Scheidt, P.C. (2003). Relationships between bullying and violence among US youth. Archives of Pediatrics \& Adolescent Medicine, 157, 348-353.

Nesdale, D., Durkin, K., Maass, A., Kiesner, J. y Griffiths, J. A. (2008). Effects of group norms on children's intentions to bully. Social Development, 17, 889-907.

OECD-FAO (2010). Perspectivas Económicas de América Latina 2010. Washington: OECD.

Ohsako, T. (1999). The developing world. En P. K. Smith, The Nature of School Bullying. A Cross-National Perspective (pp. 359-375). Londres: Routhedge.

Olweus, D. (1978). Aggression in scholls: Buliies and whipping boys. New York: Wiley.

Olweus, D. (1980). Familial and temperamental determinants of aggressive behavior in adolescent boys: A causal analysis. Developmental Psychology, 16, 644-660.

Olweus, D. (1993). Bullying at school: What we know and what we can do. New York: Blackwell.

Olweus, D. (1999). Norway. En P.K. Smith, Y. Morita, J. Junger-Tas, D. Olweus, R. Catalano y P. Slee (Eds). The nature of bullying. A cross-national perspective. London: Routledge.

Olweus, D. (2007). Acoso Escolar: hechos y medidas de intervenciones. Ponencia presentada en el Encuentro Europeo por la Convivencia. Madrid. Texto extraído de http:// www.educa.madrid.org/binary/20/DAN_OLWEUS.pdf

Olweus, D. y Endresen, I. M. (1998). The importance of sexof-stimulus object: Age trends and sex differences in empathic responsiveness. Social Development, 7, 370388.

Ortega, R. (2003). Enseñanza de prevención de la violencia en escuelas de Centroamérica (Proyecto TC/99/0011). Washington: Banco Interamericano de Desarrollo.

Ortega, R. (2008). Malos tratos entre escolares: de la investigación a la intervención. Madrid: CIDE.

Ortega, R. (Coord.) (2010). Agresividad injustificada, bullying y violencia escolar. Madrid: Alianza Editorial.

Ortega, R. y Del Rey, R. (2003). Cuestionario sobre Convivencia, Violencia y Experiencias de Riesgo - COVER. Documento no publicado.

Ortega, R. y Mora-Merchán, J. A. (2000). Violencia escolar. Mito o realidad. Sevilla: Mergablum.

Ortega, R. y Mora-Merchán J. A. (2008). Las redes de iguales y el fenómeno del acoso escolar: explorando el esquema dominio-sumisión. Infancia y Aprendizaje, 31, 515528.

Pepler, D. J., Jiang, D., Craig, W. M. y Connolly, J. (2008). Developmental trajectories of bullying and associated factors. Child Development, 79, 325-338.

Povedano, A., Hendry, L. B., Ramos M. J. y Varela, R. (2011). Victimización escolar: clima familiar, autoestima y satisfacción con la vida desde una perspectiva de género. Psychosocial Intervention, 20, 5-12. 
Rigby, K. (2002). Perspectives on bullying. London \& Philadelphia: Jessica Kingsley.

Rodkin, P. C. y Berger, C. (2008). Who bullies whom? Social status asymmetries by victim gender. International Journal of Behavioral Development, 32, 473-485.

Rutter, M., Giller, H. y Hagell, A. (2000). La conducta antisocial de los jóvenes. Madrid: Cambridge University Press.

Salmivalli, C., Lagerspetz, K. M. J., Björkqvist, K., Österman, K. y Kaukiainen, A. (1996). Bullying as a group process: participant roles and their relations to social status within the group. Aggressive Behaviour, 22, 1-15.

Schwartz, D., Dodge, K. A. y Coie, J. D. (1993). The emergence of chronic peer victimization in boys' play groups. Child Development, 64, 1755-1772.

Schwartz, D., Dodge, K. A., Pettit, G. S. y Bates, J. E. (1997). The early socialization of aggressive victims of bullying. Child Development, 68, 665-675.

Silva, L. C. y Barroso, I. M. (2004). Regresión Logística. Madrid: La Muralla.

Smith, P. K. (2009). Bullying in primary and secondary schools: Psychological and organizational comparisons. En S. Jimerson, S. Swearer y D. Espelage (Eds.), The International Handbook of School Bullying (pp. 137149). New York y Abingdon: Routledge.

Smith, P. K. y Brain, P. (2000). Bullying in schools: Lessons from two decades of research. Aggressive Behavior, 26, 1-9.

Smith, P. K., Morita, Y., Junger-Tas, J., Olweus, D., Catalano, R. y Slee, P. (Eds.) (1999). The nature of bullying. A cross national perspective. Londres: Routhedge.

Smith P. K. y Sharp, S. (1994). The problem of school bullying. En P. K. Smith y S. Sharp (Eds.), School bullying: insights and perspectives. London: Routledge.

Stein, J. A., Dukes, R. L. y Warren, J. I. (2007). Adolescent male bullies, victims, and bully-victims: A comparison of psychosocial and behavioral characteristics. Journal of Pediatric Psychology, 32, 273-282.

Tharp-Taylor, S., Haviland, A. y D'Amico, E. J. (2009). Victimization from mental and physical bullying and substance use in early adolescence. Addictive Behaviors, 34, 561-567.

Vaillancourt, T., Brendgen, M., Boivin, M. y Tremblay, R. E. (2003). A longitudinal confirmatory factor analysis of indirect and physical aggression: Evidence of two factors over time? Child Development, 74, 1628-1638.

Visauta, B. y Martori, J. (2003). Análisis Estadístico con SPSS para Windows. Madrid: McGraw-Hill.

Wang, J., Iannotti, R. J. y Nansel, T. R. (2009). School Bullying Among Adolescents in the United States: Physical, Verbal, Relational, and Cyber. Journal of Adolescent Health, 45, 368-375.

Manuscrito Recibido: 15/01/2011

Revisión Recibida: 07/02/2011

Manuscrito Aceptado: 03/04/2011 